Durch die Umfällungen ist die Menge der anderen Muskelproteine auf $<1 \%$ ihres Gehaltes im Extrakt vermindert. Die Präparation der reinen Aktomyosinlösung ist etwa 6 Stdn. nach Abschluß der Extraktion beendet.

Die Herstellung der Fäden erfolgt mit Hilfe einer Ganzglasspritze mit Glaskanüle - lichte Weite zwischen 100 und $200 \mu$. Der Myosinstrahl wird in eine rotierende Glasschale gespritzt ${ }^{20}$, die mit $\mathrm{CO}_{2}$-freiem aqua dest. von $\sim 2^{\circ} \mathrm{C}$ gefüllt ist.

Nach einem Aufenthalt von mindestens 20 min bei $0^{\circ} \mathrm{C}$ werden die Fäden in Glycerin Merck doppelt destilliert 1,23 übertragen.

Zum Gebrauch kommen die Präparate für mindestens $30 \mathrm{~min}$ in 30-proz. Glycerin, das auf $p_{\mathrm{H}} 6,7-7,0$ mit $0,01-$ $m$. Phosphat gepuffert ist.

Dann werden sie, auf ein Dynamodilatometer befestigt, über Nacht im Kühlschrank bei $\sim 3^{\circ} \mathrm{C}$ getrocknet und unter dem Mikroskop gedehnt. Spannung, Länge, Dicke und Doppelbrechung werden dabei polarisationsmikroskopisch kontrolliert.

Anschließend werden die Hebel des Dynamodilatometers mit den daran befestigten Fäden in ein Bad der Versuchslösung eingetaucht. Das Glycerin ist nach wenigen Minuten hinausdiffundiert, und der Versuch kann beginnen.

Das Mikrodynamodilatometer unterscheidet sich von der von A. Weber10 verwendeten Konstruktion nur durch schwächere Federspannung und durch das Fehlen der Klemmen zur Befestigung des Fasermodells.

20 Nach H. H. We b e r, Pflügers Arch. ges. Physiol. 235, 205 [1934].

21 Die den einzelnen Spannungen zugeordneten Längen sind in Abbildungen und Tabellen bereits auf diesen Isometriefehler korrigiert.

22 D. N oll u. H. H. W e b e r, Pflüg. Arch. ges. Physiol. 235, 234 [1934].
Die Klemmen sind durch kurze gewickelte Kunststoffhebel ersetzt, auf deren distalen waagerechten Teil die Fäden mit einem Tröpfchen Cellonlack befestigt sind. Das ist nötig, weil die Fäden so viel fragiler sind als die Glycerin-Wasser-extrahierten Muskelfasern, daß sie von den Mikroklemmen zerquetscht würden.

Die geringere Federspannung ist zweckmäßig, weil die Spannung der Fadenkontraktion kleiner ist als die Spannung der Faserkontraktion.

Infolge der mangelhaften Isometrie des Apparates verkürzt sich der Faden pro mg Spannung um $\sim 30 \mu 21$.

Die Bestimmung der Eiweißkonzentration des Einzelfadens erfolgt durch Lufttrocknung unter hygrometrischer Kontrolle der Luftfeuchtigkeit. Der nach der Trocknung noch vorhandene Wassergehalt des Fadens wird auf Grund des hygrometrischen Befundes in Rechnung gestellt mit Hilfe der identischen Quellungskurven von $\mathrm{H}$. H. W eb e r (Myosinfaden) 20 und von D. Noll und H. H. W e ber (Muskelfaser) 22.

Die $p_{\mathrm{H}}$-Kontrolle erfolgte mit der Glaselektrode.

Die Chemikalien waren analysenreine Präparate der Firma Merck, die ATP-Präparate stammen von den Firmen Hen n ing (Hamburg), R o e h m \& H a a s (Philadelphia) und S i g m a Companie (Saint Louis). Bei dem Präparat $\mathrm{R}$ o e h m \& $\mathrm{H}$ a a s handelt es sich um NaATPat in Substanz, das tiefgekühlt $\left(-20^{\circ} \mathrm{C}\right)$ aufbewahrt werden muß, bei dem Präparat von $\mathrm{S}$ igma um BaATPat. Die amerikanischen Präparate sind sehr rein: Der Gehalt von unzersetztem ATPat wurde bei allen Präparaten durch Phosphatbestimmung kontrolliert.

Für die Durchführungen dieser Kontrollen bin ich Herrn Dr. H a s s e l b a c h zu Dank verpflichtet. Herrn Institutsmechanikermeister $\mathrm{N}$ a g e $\mathrm{l}$ danke ich für die Herstellung und Pflege des Dynamodilatometers sowie einiger Hilfsapparate.

\title{
Über die Erythrocytenwerte nach einem Aderlaß bei Kaninchen mit experimenteller Polycythämie
}

Von Gerhard Ruhenstroth-Bauer

Aus dem Max-Planck-Institut für Biochemie, Tübingen

(Z. Naturforschg. 6 b, 361-365 [1951]; eingegangen am 23. August 1951)

1. Wenn Schwarzlohkaninchen durch mehrfache Injektion von Erythrocyten polycythämisch gemacht und dann einem Aderlaß unterzogen werden, so bleiben ihre Reticulocytenzahlen normal, wenn durch den Aderlaß die 5-Mill./cmm-Grenze nicht erreicht wird. Wird diese nur wenig unterschritten, so kommt es zu einer mäßigen Reticulocytose, wird sie stärker unterschritten, zu einer starken Erythropoesesteigerung.

2. Aus dieser Beobachtung folgt

a) daß ein relativer Sauerstoffmangel der spezifische Auslöser für eine Erythropoesesteigerung ist;

b) daß es sich beim erythropoetischen und blutverdünnenden stofflichen Prinzip um 2 getrennte Prinzipien handelt.

$\mathrm{W}_{\mathrm{k}}$ ird bei einem Partner einer mehrere Tage alten Kaninchen-Parabiose ein Aderlaß ausgeführt, kann man folgende 2 Veränderungen im roten Blut- bild feststellen: a) sowohl das entblutete Tier wie sein Nachbar verdünnen innerhalb Minuten bis Stunden ihr Blut, so daß eine Verminderung der Erythrocyten- 


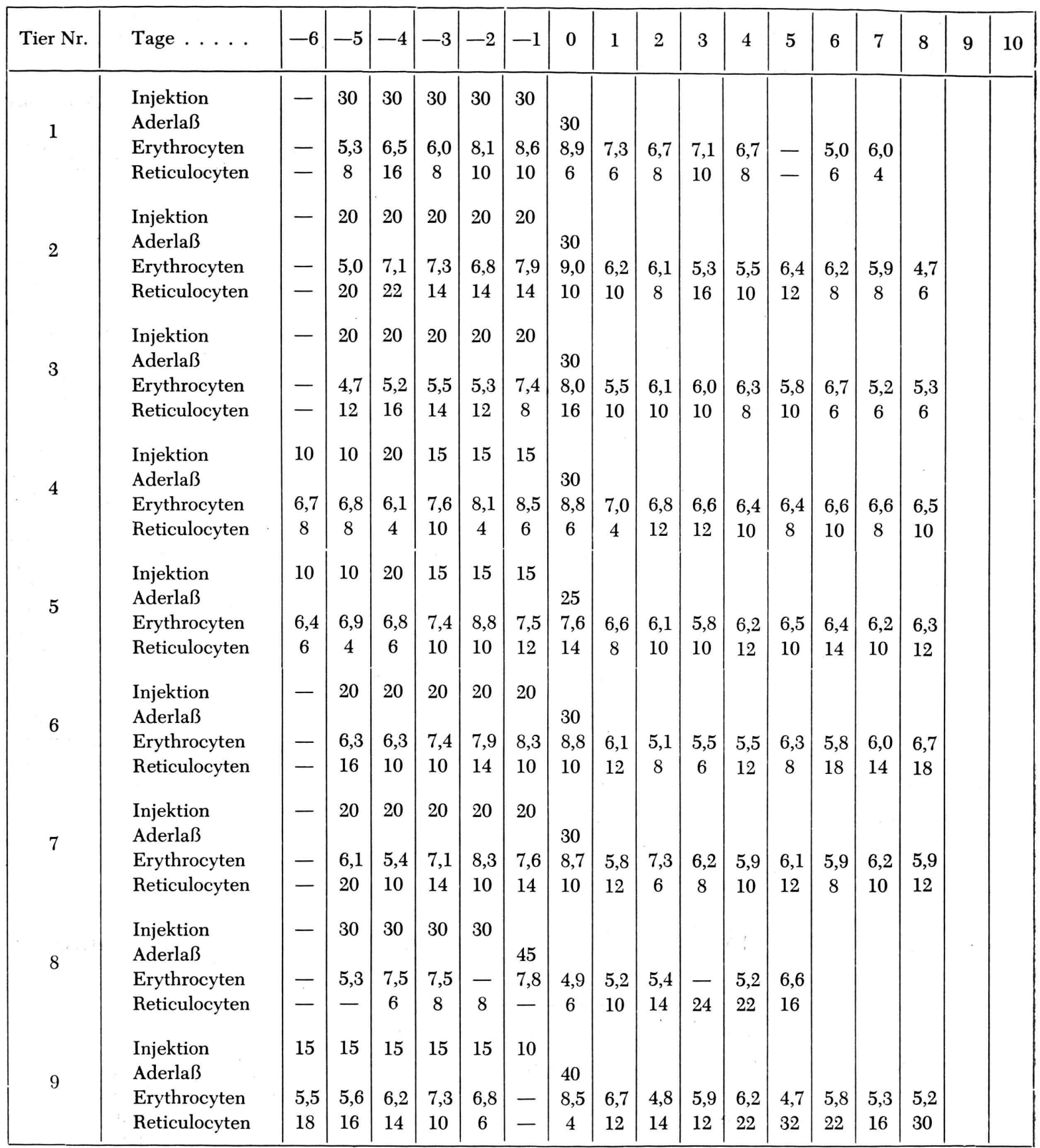

Tab. 1. Erythrocytenwerte in Mill./cmm und Reticulocytenwerte in ${ }^{0} / 00$ von Kaninchen, die nach mehrfacher Injektion $(\mathrm{cm})$ von Erythrocyten einem Aderlaß $(\mathrm{ccm})$ am Tage 0 unterzogen wurden. Die Blutwerte wurden jeweils vor den Injektionen und dem Aderlaß gezählt.

konzentration eintritt ${ }^{1}$; b) nach mehreren Tagen steigen sowohl bei dem entbluteten Kaninchen als auch bei seinem Partner die Reticulocytenwerte an, um 1 i G. Ruhenstroth-Bauer u. H. Teipel, Z. ges. exp. Med., im Druck. nach einiger Zeit wiederum zur Norm abzufallen 2 Die erste Beobachtung wurde so gedeutet, daß die Blutverdünnung durch ein stoffliches Prinzip hervor-

2 G. Ruhenstroth-B a u er, Arch. exp. Pathol. Pharmakol. 211, 32 [1950]. 


\begin{tabular}{|c|c|c|c|c|c|c|c|c|c|c|c|c|c|c|c|c|c|c|}
\hline Tier Nr. & Tage ..... & -6 & -5 & -4 & -3 & -2 & -1 & $\mathbf{0}$ & 1 & 2 & 3 & 4 & 5 & 6 & 7 & 8 & 9 & 10 \\
\hline \multirow{3}{*}{10} & Injektion & 15 & 15 & 15 & 15 & 15 & 15 & & & & & & & & & & & \\
\hline & $\begin{array}{l}\text { Aderlaß } \\
\text { Erythrociten }\end{array}$ & 47 & 59 & 65 & 74 & 77 & . & 40 & 47 & 46 & 43 & 46 & 43 & 43 & 45 & & & \\
\hline & Reticulocyten & 14 & 12 & 14 & 6 & 8 & - & 2 & 10 & 14 & 16 & 20 & 30 & 38 & 30 & & & \\
\hline \multirow{4}{*}{11} & Injektion & 15 & 15 & 15 & 15 & 15 & 15 & & & & & & & & & & & \\
\hline & Aderlaß & & & & & & & 40 & & & & & & & & & & \\
\hline & Erythrocyten & 5,5 & 5,1 & 6,9 & 6,5 & 8,3 & - & 6,9 & 5,3 & 5,4 & 5,0 & 4,7 & 5,2 & 4,3 & 4,4 & 4,6 & & \\
\hline & Reticulocyten & 8 & 8 & 6 & 8 & 6 & - & 6 & 16 & 14 & 12 & 18 & 24 & 20 & 26 & 22 & & \\
\hline \multirow{3}{*}{12} & Injektion & - & 20 & 20 & 20 & 20 & 20 & & & & & & & & & & & \\
\hline & $\begin{array}{l}\text { Aderlaß } \\
\text { Frythrocyten }\end{array}$ & - & & 64 & & 70 & 81 & 40 & 53 & 40 & 49 & 47 & 56 & & & & & \\
\hline & Reticulocyten & - & $\begin{array}{l}0,0 \\
14\end{array}$ & 10 & 12 & 10 & 12 & 12 & 12 & 20 & 16 & 10 & $\begin{array}{l}28-10 \\
28\end{array}$ & 34 & 28 & 38 & & \\
\hline & & & & & & & & & & & & & & & & & & \\
\hline \multirow{3}{*}{13} & Injektion & - & 10 & 20 & 20 & 15 & 10 & & - & & & & & & & & & \\
\hline & Erythrocyten & - & - & 5,2 & 6,4 & 6,8 & 7,9 & 7,8 & 4,6 & 4,6 & 4,9 & 3,4 & 4,4 & 5,3 & 4,5 & 4,4 & 5,0 & \\
\hline & Reticulocyten & - & - & 8 & 16 & 10 & 12 & 8 & 10 & 30 & 22 & 40 & 66 & 44 & 36 & 36 & 48 & \\
\hline \multirow{4}{*}{14} & Injektion & - & 20 & 25 & 15 & 10 & 10 & & & & & & & & & & & \\
\hline & Aderla $\beta$ & & & & & & & 60 & & & & & & & & & & \\
\hline & Erythrocyten & - & 5,2 & 6,6 & 6,8 & 7,5 & - & 8,6 & 3,8 & 4,6 & 4,5 & 4,4 & 4,9 & 5,0 & 5,0 & 5,2 & 5,2 & \\
\hline & Reticulocyten & - & 16 & 14 & 16 & 8 & - & 4 & 10 & 26 & 50 & 74 & 62 & 72 & 50 & 52 & 20 & \\
\hline \multirow{3}{*}{15} & $\begin{array}{l}\text { Injektion } \\
\text { Aderlaß }\end{array}$ & - & 20 & 15 & 15 & 15 & 10 & 50 & & & & & & & & & & \\
\hline & Erythrocyten & - & 5,8 & 5,8 & 6,4 & 6,9 & - & 4,9 & 4,1 & 4,1 & 3,3 & 3,2 & 4,0 & 4,3 & 2,8 & 4,7 . & 4,9 & \\
\hline & Reticulocyten & - & 16 & 16 & 10 & 10 & - & 6 & 10 & 30 & 60 & 122 & 112 & 150 & 106 & 56 & 30 & \\
\hline \multirow{3}{*}{16} & $\begin{array}{l}\text { Injektion } \\
\text { Aderlaß }\end{array}$ & - & 20 & 20 & 15 & 10 & 10 & 55 & & & & & & & & & & \\
\hline & Erythrocyten & - & 5,4 & 6,5 & 7,2 & 7,8 & - & 8,0 & 5,3 & 3,9 & 4,1 & 3,7 & 4,1 & 5,4 & 4,3 & 4,5 & 5,1 & \\
\hline & Reticulocyten & - & 16 & 26 & 16 & 10 & - & 8 & 8 & 24 & 66 & 74 & 76 & 106 & 88 & 30 & 24 & \\
\hline \multirow{3}{*}{17} & $\begin{array}{l}\text { Injektion } \\
\text { Aderlaß }\end{array}$ & - & 20 & 20 & 20 & 20 & 20 & 30 & & & & & & & & & & \\
\hline & Erythrocyten & - & 5,8 & 6,6 & 5,9 & 6,9 & 7,5 & 9,5 & 5,5 & 5,6 & 5,3 & 4,2 & 3,9 & 4,4 & 5,0 & 3,8 & 5,0 & \\
\hline & Reticulocyten & - & 10 & 12 & 10 & 14 & 10 & 8 & 10 & 16 & 20 & 26 & 34 & 50 & 56 & 34 & 42 & \\
\hline \multirow{3}{*}{18} & $\begin{array}{l}\text { Injektion } \\
\text { Aderlaß }\end{array}$ & 20 & 20 & 20 & 20 & 20 & 20 & 60 & & & & & & & & & & \\
\hline & Erythrocyten & 5,9 & 5,2 & 6,1 & 7,4 & 7,3 & 7,3 & 9,0 & 4,7 & 4,2 & 4,2 & 3,1 & 3,0 & 3,3 & 3,8 & 3,7 & 4,2 & 4,8 \\
\hline & Reticulocyten & 14 & 16 & 12 & 8 & 8 & - & 8 & 12 & 8 & 38 & 58 & 122 & 266 & 180 & 120 & 85 & 36 \\
\hline
\end{tabular}

Tab. 1 (Fortsetzung).

gerufen wird, das im entbluteten Tier entsteht und nach Durchwanderung durch die Parabiosebrücke auch im normalen Partner eine Verminderung der Zellkonzentration bewirkt. Die zweite Beobachtung stellt die experimentelle Grundlage für die Annahme eines spezifischen erythropoetischen Prinzips dar, das in entsprechender Weise wie das vorige entsteht und sich auswirkt. Die zweite Beobachtung und ihre Deutung fand inzwischen eine sehr eindrucksvolle Bestätigung: gleichzeitig mit der oben zitierten Arbeit erschien eine Mitteilung von $\mathrm{R}$ e i $\mathrm{Bm}$ a n $\mathbf{n}^{\mathbf{3}}$, der bei Rattenparabiosen zu dem gleichen Ergebnis kam. Rattenparabiosen weisen im Gegensatz zu 3 K. R. Reißmann, Blocd 5, 372 [1950]. 
Kaninchenparabiosen ${ }^{4}$ eine relativ starke Blutzelldurchgängigkeit auf ${ }^{5}$. Deshalb sind bei ihnen die Reticulocytenwerte als Test für ein erythropoetisches Prinzip nicht brauchbar. Aus diesem Grunde verwandte Reißmann als Test die Erythropoeseaktivität des Knochenmarks von Rattenparabiosen, von denen 1 Tier ein sauerstoffarmes Luftgemisch einatmete; bei beiden Tieren kam es zu einer statistisch gesicherten Zunahme der Knochenmarksaktivität. Zugleich konnte Reißmann ausschließen, daß die Erhöhung beim normalen Partner durch eine übertragene Verminderung der Sauerstoffsättigung des Blutes eingetreten sein konnte, denn dessen Sauerstoffsättigung war normal. So kommt auch Reißmann zu dem Schluß, daß ein stoffliches Prinzip existiert, das bei Sauerstoffmangel $\mathrm{zu}$ einer Erythropoesesteigerung führt. - Das erythropoetische Prinzip ist Glied einer Wirkkette, deren Ausgangspunkt ein „relativer Sauerstoffmangel“ ist ${ }^{2}$, d. h. ein Sauerstoffangebot, das zwar den Bedarf des Körpers an Sauerstoff deckt, aber gegenüber der Norm vermindert ist. Die vorliegende Arbeit soll einen weiteren Beleg für diesen Auslöser bringen; darüber hinaus soll gezeigt werden, daß die beiden angeführten stofflichen Prinzipien getrennt nebeneinander existieren.

\section{Methodik}

Die Versuche wurden an durchschnittlich $2 \mathrm{~kg}$ schweren Schwarzlohkaninchen ausgeführt. Über die Reinrassigkeit dieser Tiere gilt das in ${ }^{6}$ Angeführte. Die Haltung der Tiere und die Bestimmung ihrer Blutwerte entsprach den früher ${ }^{2}$ gemachten Angaben. Den Tieren wurden während 5 bis 6 Tagen Kaninchen-Erythrocyten injiziert, die aus 10 bis $20 \mathrm{ccm}$ Blut stammten. Sie wurden vor der Injektion 3-mal mit physiologischer Kochsalzlösung gewaschen. Die Gerinnung des Blutes wurde durch Heparin („Vetren“ der Firma Promonta) verhindert. Hatten die Tiere einen Erythrocytenspiegel von etwa $8 \mathrm{Mill} / \mathrm{cmm}$ erreicht, wurde mittels der Zahnschen Saugglocke 2 am Ohr ein Aderlaß von 25 bis $60 \mathrm{ccm}$ Blut ausgeführt. Während der Injektionsperiode und 1 Woche nach dem Aderla $\beta$ wurden täglich die Erythrocyten und Reticulocyten gezählt. Die Menge des injizierten und entnommenen Blutes und die Blutwerte zeigt Tab. 1.

\section{Ergebnis und Besprechung}

Aus Tab. 1 ist zu entnehmen, daß die Versuche in 3 Gruppen eingeteilt werden können:

I. In die erste Gruppe (Tier 1 bis 7 der Tab. 1) gehören alle Versuche, bei denen der Aderlaß eine Blut-

4 G. Ru he n s tr o th-B a u e r, Habilitationsschrift, Tübingen 1951.

5 D. C. van Dyke, R. L. Huff, u. H. M. Evans, Stanford M. Bull. 6, 273 [1948]. verdünnung im Gefolge hat, die die 5-Mill./cmmGrenze nicht unterschreitet. Bei keinem dieser Tiere kommt es nach dem Aderlaß zu einer Steigerung der normalen Reticulocytenzahlen $(\% / 00)$. Es sei erwähnt, daß alle Tiere auch dieser Gruppe wenige Minuten nach dem Aderlaß in ihren Erythrocytenwerten so wie normale Tiere um 1 bis 2 Millionen fielen.

II. Bei den Tieren der 2. Gruppe (Tier 8 bis 12) wird die 5-Mill./cmm-Grenze nach dem Aderlaß für kürzere Zeit um $0,1-0,7$ Mill./cmm unterschritten. Bei diesen Tieren steigen die Reticulocytenzahlen nach dem Aderlaß auf $24-38 \%$ an.

III. In der dritten Gruppe (Tier 13 bis 18) befinden sich jene Tiere, bei denen der Aderlaß eine starke $(1,2-2,2 \mathrm{Mill} . / \mathrm{cmm})$ und teilweise auch länger dauernde Unterschreitung der 5-Mill./cmm-Grenze zur Folge hat. Bei diesen Tieren ist eine Reticulocytose von $66-266^{\circ} \%$ zu beobachten.

Es sei erwähnt, daß bei 10 Vorversuchen, bei denen Kaninchen verschieden große Mengen Blut durch wechselnde Zeiträume hindurch injiziert worden waren, grundsätzlich das gleiche Ergebnis zur Beobachtung kam. Da bei Schwarzlohkaninchen die normale Erythrocytenzahl etwa $5 \mathrm{Mill} / \mathrm{cmm}$ beträgt, zeigt dieses experimentelle Ergebnis erneut ${ }^{2}$, daß eine Verminderung der Erythrocytenzahl unter die normale Konzentration die normale Erythropoese steigert. Im Gegensatz dazu wird jedoch, wie aus der Tabelle zu ersehen ist, die Erythropoese durch eine Vermehrung der normalen Erythrocytenzahlen kaum gesenkt, denn die Retikulocyten bleiben noch normal. $\mathrm{Ob}$ die gegenteiligen Erfahrungen Roberts ons ${ }^{\text {* }}$ auf die teilweise andersartige Versuchsanordnung zurückzuführen sind, ist schwer zu entscheiden.

Bei der Gruppe II kommt es nur zu einer relativ geringen Unterschreitung der 5-Mill.-Grenze, trotzdem reagieren diese Tiere mit einer Vermehrung der Reticulocytenzahlen. Diese Beobachtung ist deshalb besonders überraschend, da schon $\mathrm{B}$ a r $\mathrm{cr} \mathrm{of} \mathrm{t}^{8}$ gezeigt hat, daß das normale Sauerstoffangebot etwa vierfach größer ist als der normale Bedarf. Aus diesem Grunde kann man bei der relativ geringen Erythrocytenverminderung insbesondere dieser Gruppe nicht von „Sauerstoffmangel“" schlechthin als Auslöser der Erythropoesesteigerung sprechen, sondern nur vom „relativen Sauerstoffmangel“ im oben definier-

6 G. Ruhenstroth-B a er u. K. H. Maier, Arch. exp. Pathol. Pharmakol., im Druck.

7 O. H. Roberts o n, J. exp. Med. 26, 221 [1917].

8 J. B a r c r o f t, Erfahrungen in großen Höhen, Springer, Berlin 1927. 
ten Sinn. Es handelt sich hier offenbar um einen Parallelfall zum "hypoxischen Paradoxon“ bei der Versorgung des Hirngewebes mit Sauerstoff ${ }^{9}$.

Es sei noch auf folgende Tatsache hingewiesen: bei der III. Gruppe, bei der der stärkste Aderlaß ausgeführt worden war, ist die Blutmenge des Aderlasses noch immer geringer als diejenige aller vorher verabreichten Blutinjektionen zusammen, selbst wenn man eine übernormale Abbauquote von $8 \%$ je Tag des injizierten Blutes mit berücksichtigt; trotzdem fallen die Erythrocytenwerte nach dem Aderlaß unter die Norm. Dies kann m. E. nur so gedeutet werden: bei der Injektion größerer Mengen von Erythrocyten kommt es, wie schon länger bekannt ist ${ }^{10}$, zu einer Eröffnung von zusätzlichen Kapillargebieten, die normalerweise nicht durchströmt werden. Offenbar bleibt nach einem Aderlaß, der aus dem Blut weniger Erythrocyten entfernt, als vorher injiziert worden war, ein Teil dieser Kapillaren offen, so daß durch die Blutverdünnung eine Unterschreitung der normalen 5-Mill.-Grenze bewirkt wird. Im Organismus solcher

9 E. Opitz u. N. S chneider, Ergebn. Physiol. 46, 126 [1950].

10 D. Grepp u. C. Wiggers, Amer. J. Physiol. 104, 423 [1933].
Tiere befindet sich demnach eine größere Gesamtzahl von Erythrocyten, als der Norm entspricht, bei einer verminderten Zellkonzentration. $\mathrm{Da}$ in diesen Fällen eine kxäftige Erythropoesesteigerung beobachtet wird, läßt sich folgern, daß es bei der Steuerung der Erythropoese im normalen Organismus und bei normaler Luftatmung nicht auf die Gesamtzahl der Sauerstoffträger, sondern auf ihre Konzentration in der Volumeneinheit ankommt.

Da ein kräftiger Aderlaß, der die Erythrocytenzahlen jedoch nicht unter die 5-Mill./cmm-Grenze fallen läßt (Gruppe I), keine Steigerung der Erythropoeseaktivität mit sich bringt, sondern erst ein solcher, bei dem die Erythrocyten diese Grenze unterschreiten, läßt sich folgern, daß eine Verdünnung der Erythrocytenkonzentration als solche noch keine Steigerung der Erythropoese bewirken kann. Erst ein zusätzlicher relativer Sauerstoffmangel setzt den Erythropoesemechanismus in Bewegung. Damit scheint bewiesen, daß es sich bei der Steuerung der 2 Mechanismen um 2 getrennte stoffliche Prinzipien handeln muß.

Frl. H. T e i pe l danke ich für ihre wertvolle Hilfe bei der Durchführung der Arbeit.

\title{
Zur Kinetik des Zerfalls \\ von Diazoessigester und Nitrosoalkyl-Harnstoffen an sauren bzw. basischen Wirkungsgruppen von ionenaustauschenden Permutoiden
}

\author{
Von W. Lautsch und W. Rothkegel \\ Aus dem Institut für organische Chemie der Freien Universität Berlin, Berlin-Dahlem \\ (Z. Naturforschg. 6 b, 365-369 [1951]; eingegangen am 9. Juli 1951)
}

\begin{abstract}
Kationenaustauscher und mit quartären Ammoniumgruppen ausgestattete Permutoide bewirken den Zerfall von Diazoessigester bzw. Nitrosomethylharnstoff. Eine Abhängigkeit der Zerfallsgeschwindigkeit konnte bei geringen Austauschermengen festgestellt werden, bei größeren Austauschermengen war jedoch eine Annäherung der Zerfallsgeschwindigkeitskonstanten an einen Grenzwert zu bemerken. Diese Beobachtungen können für Austauschersäulen von Interesse sein, da dort annähernd gleiche Versuchsbedingungen vorliegen.
\end{abstract}

1. Die katalytische Wirksamkeit von mit Sulfons äure-bzw. Carboxylgruppen ausgerüsteten Kationen-Austauschern auf den Zerfall von Diazoessigester

$\mathrm{V}$ or mehr als 40 Jahren zeigte B redig ${ }^{1}$, daß der Zerfall von Diazoessigester unter Bildung von Stickstoff und Glykolsäureester „zu den empfindlichsten Katalysen durch Wasserstoffionen gehört" und daß diese Reaktion nach einem Zeitgesetz 1. Ordnung verläuft.

Wir verwendeten diese Reaktion in der Absicht, eine Aussage machen zu können über die katalytische Wirksamkeit der Wasserstoffionen, die in den $\mathrm{SO}_{3} \mathrm{H}$ und $\mathrm{COOH}-G r u p p e n$ der Ionen-Austauscher enthalten sind.

1 G. B re dig u. W. Fränke l, Z. Elektrochem. angew. physik. Chem. 11, 525 [1905]. 